\title{
Silicon slow-light-based photonic mixer for microwave-frequency conversion applications
}

\author{
A. M. Gutiérrez, ${ }^{1, *}$ A. Brimont, ${ }^{1}$ J. Herrera, ${ }^{2}$ M. Aamer, ${ }^{1}$ J. Martí, ${ }^{1}$ D. J. Thomson, ${ }^{3,4}$ F. Y. Gardes,,${ }^{3,4}$ \\ G. T. Reed,,$^{3,4}$ J. M. Fedeli, ${ }^{5}$ and P. Sanchis ${ }^{1}$ \\ ${ }^{1}$ Nanophotonics Technology Center, Universitat Politecnica Valencia, Camino de Vera s/n, 46022, Valencia, Spain \\ ${ }^{2}$ Fibernova Systems, Universitat Politecnica Valencia, Camino de Vera s/n, 46022, Valencia, Spain \\ ${ }^{3}$ School of Electronics and Computer Science, University of Southampton, Southampton, UK \\ ${ }^{4}$ Some work perfomed while at the University of Surrey, Guildford, Surrey Gu2 7XH, UK \\ ${ }^{5}$ CEA, LETI, Minatec Campus, 17 Rue des Martyrs, 38054, Grenoble CEDEX, France \\ ${ }^{*}$ Corresponding author: angucam@ntc.upv.es
}

Received February 9, 2012; revised March 21, 2012; accepted March 23, 2012;

posted March 23, 2012 (Doc. ID 162063); published May 14, 2012

\begin{abstract}
We describe and demonstrate experimentally a method for photonic mixing of microwave signals by using a silicon electro-optical Mach-Zehnder modulator enhanced via slow-light propagation. Slow light with a group index of $\sim 11$, achieved in a one-dimensional periodic structure, is exploited to improve the upconversion performance of an input frequency signal from 1 to $10.25 \mathrm{GHz}$. A minimum transmission point is used to successfully demonstrate the upconversion with very low conversion losses of $\sim 7 \mathrm{~dB}$ and excellent quality of the received $I / Q$ modulated QPSK signal with an optimum EVM of $\sim 8 \%$. (C) 2012 Optical Society of America

OCIS codes: $130.3120,190.2620,190.7220,250.4110$.
\end{abstract}

Radio-frequency (RF) analog data processing in analog photonic links encompasses numerous potentially rewarding applications such as radio-over-fiber signal transmission, photonic analog-to-digital conversion, antenna remoting, or microwave/millimeter-wave generation [1]. The use of silicon photonic devices to transmit and process analog RF signals may lead to chip-size reduction and power-efficiency improvements. When signal frequencies are in the microwave/millimeter-wave bands, light is commonly modulated using external electro-optical modulation. The plasma dispersion effect is currently considered one of the leading approaches for developing electro-optical modulators in silicon photonics. Significant milestones have been reported during recent years using this effect for digital communications [2]. However, very little attention has been paid to analog links compared to the digital ones. High linearity is a typical requirement for analog signals, and therefore modulators are operated in the linear regime. Such modulation linearity has been theoretically analyzed for silicon Mach-Zehnder modulators (MZM) [3] as well as silicon microring-based modulators [4]. Furthermore, a silicon MZM has also been successfully employed to transmit an 802.11 OFDM signal [5]. However, in addition to the plain analog signal transmission, nonlinear applications such as RF mixing techniques have also attracted a strong interest. In contrast with the transmission, these mixing techniques exploit the nonlinear operation of the electro-optical modulator. Photonic mixing techniques for analog links feature superior characteristics such as ultrawide bandwidth and immunity to electromagnetic interference when compared with conventional RF mixing schemes. Several works have reported photonic mixers using commercial $\mathrm{LiNbO}_{3}$ modulators [6,7]. However, to the best of our knowledge, no work on silicon photonic mixers has been demonstrated so far. On the other hand, the use of slow-light propagation is a well-known approach to enhance modulation efficiency [8]. In the case of silicon, the nonlinear phase response intrinsic to free carrier dispersion has been shown to be dependent on the optical group index [도요. Therefore, this dependence can be further exploited to enhance the conversion efficiency of the mixing process.

In this work, we experimentally demonstrate a siliconbased photonic mixer for up-frequency conversion. A silicon-based MZM enhanced via slow-light propagation (Fig. 1) is used to generate the mixing signal. Device performance is measured in terms of spurious-free dynamic range (SFDR) and conversion loss. Also, the error vector magnitude (EVM) is measured for the mixed received signal using a quadrature phase-shifting keying (QPSK) modulation format. Furthermore, comparisons between the slow- and fast-light regions and optimization of the operating bias point have been carried out.

Slow-light propagation is achieved through the use of a one-dimensional periodic structure consisting of a laterally corrugated waveguide. Optical phase is modulated by depleting the majority carriers from a reverse-biased $p n$ junction connected to highly doped $n+$ and $p+$ regions. Details of doping conditions and parameters of

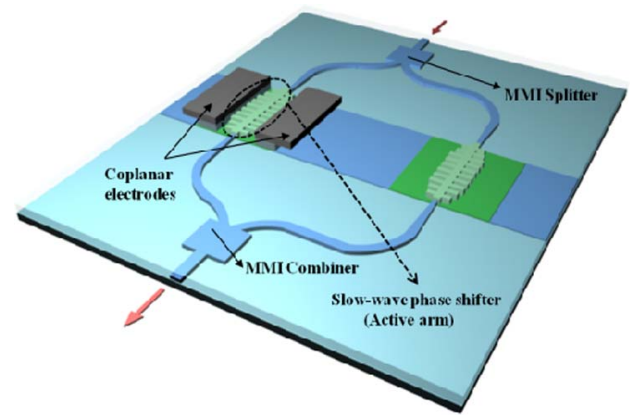

Fig. 1. (Color online) Schematic of the slow-wave modulator used for mixing. 
the slow-light structure can be found in [9]. Optical phase modulation in the slow-wave waveguide is converted into amplitude modulation via the use of an asymmetric Mach-Zehnder interferometer (MZI) with a slow-light phase-shifter length of $500 \mu \mathrm{m}$ and a length difference between two arms of $900 \mu \mathrm{m}$. As demonstrated in [9] for digital measurements for such MZM, the slow-light effect enhanced the modulation efficiency, allowing modulation data rates up to $40 \mathrm{~Gb} / \mathrm{s}$ in such a short length.

In the experimental setup shown in Fig. 2, the input light emitted by an external cavity laser is coupled from standard single-mode fibers to the chip via grating couplers. Two signal generators operating at $\left(f_{\mathrm{IF}}\right)$ and $\left(f_{\mathrm{OL}}\right)$ are power combined and the two-tone input is coupled to a DC bias using a bias tee and applied through high-speed ground-signal-ground probes to one port of the slow-light MZM (the other port is terminated with a $50 \Omega$ load). The output-modulated signal is amplified, filtered, and photo detected by a high-speed photodiode. Finally, a vectorial signal analyzer is used to measure the received signal. Electrical amplification is performed with a noise amplifier featuring $26 \mathrm{~dB} \mathrm{RF}$ gain and $6 \mathrm{~dB}$ noise figure.

To study the mixing efficiency, a two-tone test procedure has been carried out, as typically performed in $\mathrm{RF}$ mixers. The IF and OL frequencies are set to 1 and $9.25 \mathrm{GHz}$, respectively. Mixing is achieved by exploiting the modulator nonlinearity, which produces several harmonics and intermodulation products at different frequencies. Nonlinearity depends basically on the operating bias point of the MZI transfer function but also on the intrinsic nonlinear response to the material in the active region. In the case of $\mathrm{LiNbO}_{3}$ modulators, the former is the dominant contribution to the nonlinear performance. Therefore, the quadrature bias (QB) point, corresponding to a phase shift of $\pi / 2$ between the arms of the MZI, is usually exploited to convert the IF frequency to the third-order intermodulation distortion (IMD3) products $\left(2 f_{\mathrm{IF}} \pm f_{\mathrm{OL}}, 2 f_{\mathrm{OL}} \pm f_{\mathrm{IF}}\right)$ while the second-order intermodulation products $\left(f_{\mathrm{IF}} \pm f_{\mathrm{OL}}\right)$ are minimized. However, in our case, the nonlinear response of the free-carrier dispersion in silicon together with the slow-light propagation also plays a prominent role in the mixing performance. It must be stated that, due to the asymmetric MZI structure used here, the bias point can be tuned either through acting on the DC voltage or on the operation wavelength. In our case, the experimental measurements have been carried out by utilizing a fixed $2 \mathrm{~V}$ DC voltage and tuning the wavelength to drive the modulator to the appropriate bias operating point. In

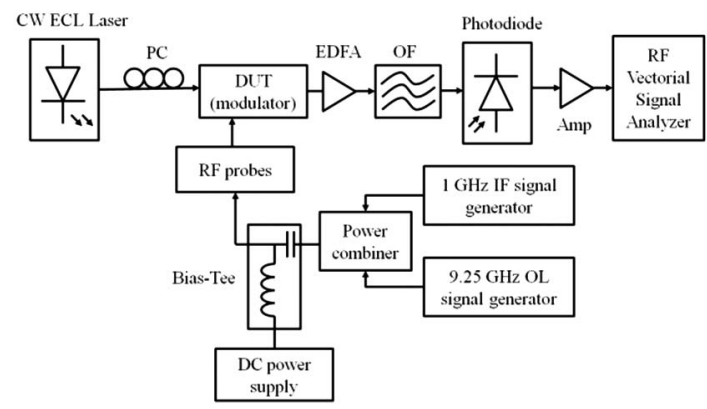

Fig. 2. Schematic of the experimental setup. this way, we can distinguish the operating wavelength in the slow-light region $(\lambda \sim 1551 \mathrm{~nm})$, where the measured group index is around 11, from the operating wavelength in the fast-light region $(\lambda \sim 1559 \mathrm{~nm})$, where the measured group index is around 4.5.

To evaluate the mixing performance of our silicon modulator, we have defined the conversion losses as the ratio between the photo-detected electrical power at $f_{\text {IF }}$ and the photo-detected electrical power of the considered intermodulation term at $N f_{\text {IF }}+f_{\text {OL }}(N=1$ for IMD2 and $N=2$ for IMD3), as in [6]. According to this definition, when a given intermodulation product power is maximized with the bias point, the conversion losses of the product should be minimized. Initially, we investigated the nonlinear performance and conversion losses at QB. Conversely to $\mathrm{LiNbO}_{3}$ modulators, we obtained large conversion losses (around $55 \mathrm{~dB}$ for $+15 \mathrm{dBm} \mathrm{IF}$ input power) for the IMD3 $\left(2 f_{\mathrm{IF}}+f_{\mathrm{OL}}=11.25 \mathrm{GHz}\right)$. Furthermore, lower conversion losses were measured (35 $\mathrm{dB}$ for $+15 \mathrm{dBm}$ IF input power) for the IMD2 $\left(2 f_{\mathrm{IF}}+f_{\mathrm{OL}}=10.25 \mathrm{GHz}\right)$, as shown in Fig. 3. Based on these results, the modulator was biased at minimum transmission (MITB), which corresponds to a phase shift of $\pi$ between the arms of the MZI and where the IMD2 products are usually maximized and dominate over the third order and the fundamental terms [10]. In this case, conversion losses for IMD2, shown in Fig. 3, were reduced by more than half (around $13 \mathrm{~dB}$ for $+15 \mathrm{dBm} \mathrm{IF}$ input power), and therefore a significant improvement results in conversion losses $(\sim 30 \mathrm{~dB})$ between biasing the modulator at QB or MITB. On the other hand, lower conversion losses were also obtained in the slow-light region when compared to the fast-light region $(\sim 2-4 \mathrm{~dB}$ more at MITB and $\sim 1 \mathrm{~dB}$ at $\mathrm{QB})$. As a result, the frequency $\left(f_{\mathrm{OL}}+f_{\mathrm{IF}}\right)$ and MITB operation point were chosen to upconvert a vector modulated signal.

The SFDR-defined as the range of output powers over which no intermodulation signal is above the noise level, implying that no disturbance of the desired signal is produced-has been also measured. For our mixing functionality, we consider that the signal to which we will later upconvert a modulated signal is the $f_{\mathrm{IF}}+f_{\mathrm{OL}}$ product (IMD2). Therefore, if one qualitatively observes the output frequency spectrum diagram of a mixer, it is realized that the critical combination product in that

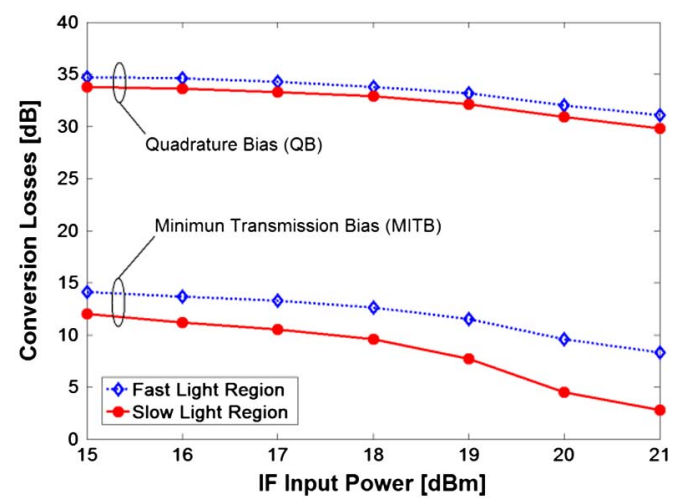

Fig. 3. (Color online) Conversion losses at QB and MITB bias points for both slow- and fast-light regions at IMD2 frequency. OL input power was set to be $+20 \mathrm{dBm}$. 


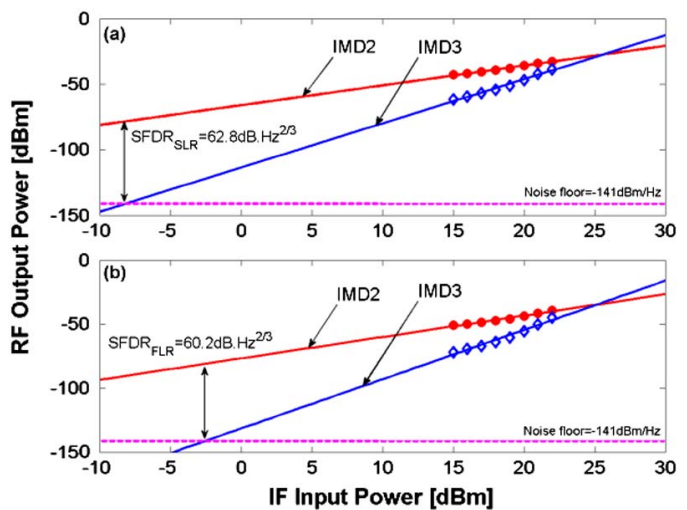

Fig. 4. (Color online) IMD2 and IMD3 powers as a function of the IF input power at MITB. SFDR for both the (a) slow- and (b) fast-light region are represented. Noise floor level has been measured at $-141 \mathrm{dBm} / \mathrm{Hz}$. OL input power was set to be $+20 \mathrm{dBm}$.

case is $2 f_{\mathrm{IF}}+f_{\mathrm{OL}}$ (IMD3) since it is the closest and therefore the most difficult to filter. As illustrated in Fig. 4, our SFDR is the output power difference between the intersection of the IMD3 power signal at $11.25 \mathrm{GHz}$ with the noise floor and the IMD2 power signal at $10.25 \mathrm{GHz}$. SFDR values of $62.8 \mathrm{~dB} . \mathrm{Hz}^{2 / 3}$ and $60.2 \mathrm{~dB} . \mathrm{Hz}^{2 / 3}$ for the slow- and fast-light regimes have been measured, respectively. For completeness, the measured intermodulation terms grow, respectively, with a slope of approximately 2 for IMD2 and 3 for IMD3, as expected from theory [10]. These results confirm again the enhanced performance brought by the slow-light regime of the modulator. In both cases, a third-order intercept point around $~ 25 \mathrm{dBm}$ has been measured.

The EVM parameter is used in wireless system standards such as IEEE $802.11 \mathrm{~g} / \mathrm{b}$, UMTS, and WiMAX to evaluate the receiver performance implementing the respective standard. For the EVM measurements and for the test scenario presented here, we choose an $I / Q$ modulated signal at the IF frequency of $f_{\mathrm{IF}}=1 \mathrm{GHz}$ with a QPSK modulation scheme with $10 \mathrm{Msymbol} / \mathrm{s}$, upconverted to a frequency of $f_{\mathrm{IF}}+f_{\mathrm{OL}}=10.25 \mathrm{GHz}$ with the electro-optic modulator biased at the MITB point.

The experimental setup is similar to that depicted in Fig. 2, with the IF signal generator and vectorial signal analyzer properly programmed for such measurements. Figure 5 shows the EVM measurements in both slowand fast-light regions. The inset depicts the received constellation at $10.25 \mathrm{GHz}$. It is clear from Fig. 5 that enhanced performance in terms of EVM is obtained in the slow-light regime over the entire range of IF input powers. Outstanding results have been obtained with a very low optimum EVM of $8 \%$ and $12 \%$ for both slow- and fast-light regimes, respectively, allowing a very clean constellation. This confirms that the received signal is of excellent quality. Furthermore, the curve meets the expected behavior and decreases for low IF input power (due to the electrical noise) since an optimum point is utilized. It should be noticed that from this optimum IF input power point $(17 \mathrm{dBm})$, the curve increases due to the IF source power saturation.

We have shown a very efficient method of generating mixing products in a silicon modulator via exploiting the

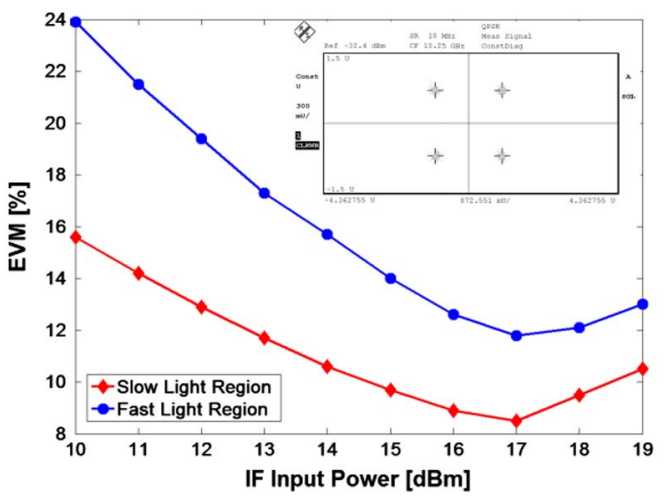

Fig. 5. (Color online) EVM as a function of IF input power for both fast- and slow-light propagation. Inset shows received constellation in the slow-light region case at the optimum point of IF input power.

nonlinearity produced by both the MZI transfer function and the plasma dispersion effect in silicon. Furthermore, the conversion efficiency has been enhanced via slowlight propagation in the active region of the modulator. Optimum performance has been achieved at MITB with low conversion losses around $7 \mathrm{~dB}$ and large SFDR of $62.8 \mathrm{~dB} . \mathrm{Hz}^{2 / 3}$. As a matter of evaluation, a $10 \mathrm{Msymbol} / \mathrm{s}$ QPSK signal at $1 \mathrm{GHz}$ frequency has been successfully upconverted to $10.25 \mathrm{GHz}$ without the need for highfrequency complex RF components. A minimum EVM of about $8 \%$ has been achieved, meeting the EVM requirements well enough for an acceptable quality of the received constellation. Overall, this simple photonic silicon slow-light-based mixer scheme is therefore a promising candidate for broadband microwave/millimeterwave communication applications.

Financial support from FP7-224312 HELIOS project and Generalitat Valenciana under PROMETEO-2010-087 R\&D Excellency Program (NANOMET) are acknowledged. F. Y.Gardes, D. J. Thomson, and G. T. Reed are supported by funding received from the UK EPSRC funding body under the grant "UK Silicon Photonics." The author A. M. Gutiérrez thanks D. Marpaung for his useful help.

\section{References}

1. J. Capmany and D. Novak, Nat. Photon. 1, 319 (2007).

2. G. T. Reed, G. Mashanovich, F. Y. Gardes, and D. J. Thomson, Nat. Photon. 4, 660 (2010).

3. A. Khilo, C. Sorace, and F. Kärtner, Opt. Express 19, 4485 (2011).

4. S. Muping, Z. Lin, R. G. Beausoleil, and A. E. Willner, IEEE J. Sel. Top. Quantum Electron. 16, 185 (2010).

5. F. Vacondio, M. Mirshafiei, J. Basak, L. Ansheng, L. Ling, M. Paniccia, and L. A. Rusch, IEEE J. Sel. Top. Quantum Electron. 16, 141 (2010).

6. J. Marti, V. Polo, F. Ramos, and J. M. Fuster, Wireless Pers. Commun. 15, 31 (2000).

7. B. Cabon, Sci. Iran. Trans. D 17, 149 (2010).

8. H. F. Taylor, J. Lightwave Technol. 17, 1875 (1999).

9. A. Brimont, D. J. Thomson, P. Sanchis, J. Herrera, F. Y. Gardes, J. M. Fedeli, G. T. Reed, and J. Martí, Opt. Express 19, 20876 (2011).

10. D. Marpaung, "High dynamic range analog photonic links design and implementation," thesis (University of Twente, Netherlands, 2009). 